notes de lecture

\title{
Vivre les valeurs du service public
}

Fabrice Larat, Christian Chauvigné (coord.)

Presses de l'EHESP-RESP (Profession cadre service public), 2016, 176 p.

Jean-Pierre Véran

\section{OpenEdition}

Journals

Édition électronique

URL : https://journals.openedition.org/ries/5592

DOI : $10.4000 /$ ries.5592

ISSN : 2261-4265

Éditeur

France Education international

Édition imprimée

Date de publication : 1 décembre 2016

Pagination : $37-38$

ISBN : 978-2-85420-612-8

ISSN : $1254-4590$

\section{Référence électronique}

Jean-Pierre Véran, «Vivre les valeurs du service public », Revue internationale d'éducation de Sèvres [En ligne], 73 | décembre 2016, mis en ligne le 01 décembre 2016, consulté le 03 juillet 2021. URL : http:// journals.openedition.org/ries/5592 ; DOI : https://doi.org/10.4000/ries.5592

Ce document a été généré automatiquement le 3 juillet 2021.

(c) Tous droits réservés 


\section{notes de lecture}

\section{Vivre les valeurs du service public}

Fabrice Larat, Christian Chauvigné (coord.)

Presses de l'EHESP-RESP (Profession cadre service public), 2016, 176 p.

\section{Jean-Pierre Véran}

Dans son allocution d'ouverture du colloque « Faire des choix ? Les fonctionnaires dans l'Europe des dictatures, 1933-1948 ", tenu à la Sorbonne, le 21 février 2013, Jean-Marc Sauvé, vice-président du Conseil d'État, affirmait :

Il n'y a pas d'État sans fonction publique, ni de fonction publique sans éthique. L'exigence éthique surplombe les techniques et méthodes de l'administration: les leçons de l'histoire nous exhortent à ne pas l'oublier aujourd'hui. Dans leur rapport au politique, à la loi, à l'autorité hiérarchique, les fonctionnaires ont une responsabilité et des devoirs particuliers qui doivent se nourrir de nos expériences, même les plus douloureuses. L'obéissance hiérarchique, qui est un principe cardinal, ne saurait échapper aux questionnements: obéir, ce n'est pas se soumettre, ni renoncer à penser, ni devoir se taire ; ce principe s'assortit même, dans des cas exceptionnels, du devoir de désobéir.

2 C'est Jean-Marc Sauvé qui préface Vivre les valeurs du service public, ouvrage coordonné par Fabrice Larat et Christian Chauvigné. Cette publication intervient dans un contexte historique bien différent de celui de l'occupation nazie, contexte marqué par «la montée en puissance des impératifs de performance et d'efficacité, la diffusion des tâches d'intérêt général hors de la sphère publique, le recul de l'État-providence, les contraintes budgétaires, l'accélération du progrès technique et la globalisation de l'économie ". C'est dire si ce contexte mondial concerne tous les pays, et on est reconnaissant aux auteurs d'avoir étayé leurs propos de travaux de droit comparé, concernant notamment le Québec et la Suisse.

3 L'ouvrage part donc de la réalité soulignée par divers acteurs des fonctions publiques territoriale, hospitalière, d'État - armée, éducation, renseignement : la confrontation entre les valeurs traditionnelles de la fonction publique et la nouvelle gestion publique inspirée du modèle du New Public Management.

4 Le socle des premières sont, en France, d'une part, les valeurs de la trilogie républicaine et le principe de laïcité, qui se traduisent par le sens de l'intérêt général, le respect de la 
diversité, l'égalité de traitement et la solidarité, et d'autre part les exigences de l'emploi public: loyauté, intégrité, neutralité, exemplarité, continuité, adaptabilité. Peuvent-elles s'enrichir ou se transformer au contact des dominantes du nouveau management public, qui sont celles de tous les secteurs de production et de service: qualité, performance, efficience ? La conciliation est-elle possible? Les situations sont variables, selon les institutions, selon les moments de la politique publique, mais aussi selon les interprétations différentes que les cadres d'une même institution peuvent avoir d'une valeur ou d'un principe. Les lectures très différentes du principe de laïcité dans un lycée par deux chefs d'établissement successifs en offrent un exemple frappant.

5 Pourtant, c'est non pas en occultant le débat, mais au contraire en l'instaurant, que le cadre responsable peut trouver un moyen de management efficace, qui redonne du sens à l'action publique, et, éventuellement, celui de résister à certaines pressions venues de sa hiérarchie, de ses agents ou des usagers de son service.

6 Les perspectives proposées par l'ouvrage sont nettes: il s'agit de "clarifier le sens, prévenir les risques éthiques, manager les valeurs", comme l'écrivent les coordonnateurs de l'ouvrage ${ }^{1}$. Elles sont enrichies des expériences conduites au Québec et en Suisse. Dans la police québécoise, Yves Boisvert ${ }^{2}$ observe que la formation sur les valeurs allant de pair avec un management éthique a permis de réduire les risques éthiques d'inconduite des agents publics. Yves Emery et David Giauque ${ }^{3}$, s'appuyant sur des enquêtes récentes portant sur les administrations publiques municipales et cantonales suisses, dégagent chez les agents «des motivations et identités qui dépendent fortement des valeurs ", même si les motivations varient fortement selon les agents. Les valeurs, ajoutent-ils, «constituent une explication non négligeable mais malheureusement souvent négligée, ou encore traitée de manière simplificatrice, des processus motivationnels et identitaires dans les organisations publiques du $\mathrm{XXI}^{\mathrm{e}}$ siècle .

7 La construction de l'ouvrage en études de cas, suivies d'éclairages ouvrant sur des perspectives, est particulièrement dynamique. On retiendra de la conclusion la nécessité d'expliciter le sens donné aux valeurs, de pratiquer une éthique collective, gage de prévention et de cohésion, nécessités qui font émerger l'ambition d'un management par les valeurs : il n'y a pas de projet de service ou d'établissement qui mobilise les acteurs sans mettre les valeurs au centre de celui-ci ; il n'y a, pour ce faire d'autre stratégie que participative, engageante, favorisant la cohésion de l'organisation autour des valeurs.

8 Cet ouvrage contribue à prolonger la réflexion conduite sur la gouvernance des organisations publiques. On connait deux dimensions possibles de cette gouvernance, qu'Alain Bouvier a caractérisées ainsi ${ }^{4}$ : la dimension instrumentale, qui vise prioritairement l'efficacité de l'action publique, et la dimension procédurale, qui se préoccupe d'accorder un rôle nouveaux aux citoyens, groupes, communautés que les Québécois désignent sous le terme d'« empouvoirement ». L'ouvrage incite à y ajouter une dimension éthique, qui n'a rien d'une utopie. Les résultats récemment publiés de l'enquête PISA 2015 de l'OCDE attestent une nouvelle fois que l'école française ne tient pas du tout sa promesse d'égalité des chances, puisque les parcours scolaires y sont, plus fortement que dans d'autres pays, corrélés à l'origine sociale des élèves. Il existe donc des indicateurs utiles pour évaluer la mise en œuvre effective à l'école de la valeur républicaine d'égalité. Il en va de même pour les données sur le climat scolaire: si 
environ $30 \%$ des élèves se sentent en situation d'injustice dans le système scolaire français, selon Marie Duru-Bellat et Denis Meuret $^{5}$, c'est sans doute aussi que la fraternité n'y est pas souveraine. Plus qu'un horizon d'attente, le management par les valeurs est donc une exigence.

\section{NOTES DE FIN}

1. C. Chauvigné, psychosociologue, et F. Larat, École nationale d'administration.

2. Professeur à l'École nationale d'administration publique du Québec.

3. Professeur de management public et gestion des ressources humaines et professeur de sociologie des organisations et des administrations publiques à l'Université de Lausanne.

4. A. Bouvier (2007) : La gouvernance des systèmes éducatifs, PUF, (Politique d'aujourd'hui).

5. M. Duru-Bellat et D. Meuret (2010) : Les sentiments de justice à et sur l'école, Bruxelles, De Boeck.

\section{AUTEUR}

\section{JEAN-PIERRE VÉRAN}

Jean-Pierre Véran est inspecteur d'académie (H). Il intervient en formation de l'encadrement en académie et à l'École supérieure de l'éducation nationale (ESEN) sur la gouvernance des organisations éducatives, les politiques éducatives et l'éducation aux médias et à l'information. Courriel : jeanpierreveran@gmail.com. Blog : http://blogs.mediapart.fr/blog/jean-pierre-veran/ 